\title{
Optimización de Layout de Planta, Síntesis y Topografía de Playas de Almacenamiento
}

\section{Optimization of Plant Layout, Tank Farm Synthesis and Topography.}

Presentación: 10/10/2019

\section{Doctorando:}

\section{Santiago Orellano}

CAIMI (Centro de Aplicaciones Informáticas y Modelado en Ingeniería), Universidad Tecnológica Nacional (FRRo). sorellano@frro.utn.edu.ar

\section{Directores: \\ Nicolás José Scenna \\ Néstor Hugo Rodríguez}

\section{Resumen}

A través del presente trabajo se presentan modelos de programación mixta entera lineal (MILP), con el objetivo optimizar por un lado la síntesis de la playa de almacenamiento de sustancias peligrosas (en este caso inflamables) considerando la posibilidad de incendio de trinchera (trench fire) y por otro, la distribución del layout general de planta donde dicha playa se integra. Se incluye además la adaptación y vinculación de ambos modelos a través de su ejecución secuencial, pretendiéndose la minimización de los costos asociados al diseño del layout y a la playa de almacenamiento contemplando el riesgo.

Se incluye además la aplicación de esta metodología y los modelos a un caso de estudio particular, la optimización del layout de una planta de biodiesel con una producción de 720 Ton/día incluyendo la optimización rigurosa de la playa de tanques de metanol.

Palabras claves: Layout de Planta, Almacenamiento de Sustancias Combustibles, Seguridad de Procesos, MILP.

\section{Abstract}

- Mixed Integer Linear Program (MILP) models are proposed in this work with the objective of optimize both, the dangerous substances tank farm synthesis (in this case flammable) considering the possibility of trench fire and the general plant layout where this farm is included. It's also included the adaptation and connection between both models using a sequential methodology. Through this, it's pretended to minimize the costs associate to the general layout design and to the tanks farm considering risks.

This methodology with the models is applied to a particular case of study that is included here. It's a biodiesel plant layout optimization with a production of 720 tons per day including the rigorous optimization of the methanol tank farm considering the trench fire possibility.

Keywords: Plant Layout, Fuel Tank Farm, Process Plant Safety, MILP.

\section{Introducción}

La mayoría de las investigaciones previas en el campo del diseño de la distribución de planta generalmente se basaban en experiencias personales. En las últimas décadas, se publicaron diversos artículos que intentaron resolver el problema del layout a través de programación matemática y de la aplicación de técnicas de optimización. Surgieron entonces, dos corrientes bien diferenciadas. Por un lado, se utilizaron métodos determinísticos de optimización (con la limitación de la cantidad de unidades de proceso posibles a tratar) y por otro, se aplicaron técnicas de optimización heurísticas (Genetics algorithm, Bat Methaheuristics algorithm, Tabu Search, Simulated annealing, entre otras), con la limitación de no poder garantizar los atributos de la solución óptima obtenida.

Es importante remarcar que, en las últimas décadas, se ha impuesto considerar la sostenibilidad de los procesos; lo cual, entre otros factores, implica seguridad operativa y de diseño de los mismos. Entre los enfoques más 
importantes y consolidados en las últimas dos décadas podemos citar las estrategias de diseño utilizadas para la incorporación de la filosofía de diseño inherentemente seguro o bien de diseño basado en riesgo.

Entre los principios del diseño inherentemente seguro se hace especial énfasis en la minimización de los inventarios de sustancias con cierto riesgo asociado. Casal (2008) realiza un análisis histórico de los accidentes en la industria, determinando que el $17 \%$ de los mayores accidentes en la industria de proceso ocurrieron o involucraron a las instalaciones de almacenamiento. Incluso la NFPA (Nacional Fire and Protection Association de los Estados Unidos) reportó que en 2009 el $13 \%$ de los mayores incendios accidentales ocurrieron en tanques de almacenamiento. Estos números reflejan el riesgo asociado a las playas de almacenamiento, y por lo tanto la necesidad de un tratamiento adecuado. Sin embargo, existen muy pocos trabajos orientados a la determinación de la estructura de la playa de almacenamiento considerando el diseño basado en riesgos; y menos aún que intenten vincular este diseño con modelos de optimización de layout, cuando en realidad son problemas estrechamente relacionados. En general, la vinculación entre etapas tempranas del diseño de procesos y la optimización de layout es un campo de investigación poco explorado y muy prometedor.

A través de este trabajo se presentan los avances logrados en el marco de la tesis doctoral "Metodología para la Optimización de Layout y Síntesis de Procesos Considerando el Riesgo Tecnológico". Se aborda la problemática de integrar el problema de minimización del riesgo inherente a las playas de almacenamiento de sustancias peligrosas (por ejemplo, combustibles) con el problema de la optimización del layout de planta. Se parte para ello de dos modelos de optimización determinísticos: por un lado, un modelo mixto entero lineal (MILP) que permite determinar el número óptimo de tanques, su distribución en el espacio, la forma de construcción del endicamiento (decisiones acerca de la construcción de paredes divisoras del dique), entre otras (Orellano et al., 2019 a) y, por otra parte, una estrategia de optimización del layout de planta en base a programación matemática (Orellano et al., 2018). Se incluye la estrategia de resolución utilizada en Orellano et al., (2019 b).

\section{Desarrollo}

Se propone una estrategia de resolución secuencial. La primera etapa consiste en la optimización del layout de la playa de tanques. Dada esta configuración, se optimizan en una segunda etapa los distintos sectores del complejo industrial, donde se incluye la disposición de tanques óptima obtenida en la etapa anterior.

En el modelo de optimización de síntesis de la playa se considera, además de los costos directamente vinculados con la misma, el costo del área exterior potencialmente afectada debido a la ocurrencia de un accidente (en este caso el área sujeta a una radiación superior a $15 \mathrm{Kw} / \mathrm{m}^{2}$ frente a la ocurrencia de un trench fire). Este flujo calorífico se establece considerando el límite propuesto por Landucci et al. (2009) para evitar la ocurrencia de efecto dominó. Se compatibilizan de esta manera las funciones objetivo utilizadas en ambas etapas secuenciales de resolución.

\section{Caso de Estudio}

Se propone a modo ejemplificador de los modelos y de la metodología utilizada el diseño del layout general de una planta de biodiesel. La misma tiene especificada como objetivo de diseño una producción de 720 Ton/día. Dada la estructura del proceso y los parámetros de operación de la planta, es posible simular la misma, y obtener mediante resolución de los balances de materia y energía los consumos de materias primas y producción de subproductos. Estableciendo un inventario para 7 días de producción, se adoptan los siguientes volúmenes de almacenamiento: aceite: $5740 \mathrm{~m}^{3}$, metanol: $1000 \mathrm{~m}^{3}$, glicerina: $1000 \mathrm{~m}^{3}$, biodiesel: $5740 \mathrm{~m}^{3}$.

Con respecto a la presencia de calles de circulación interna o pasajes peatonales, se podrían considerar distintas alternativas -ver Orellano et al. (2018)- o bien establecerse distanciamientos mínimos entre los diferentes sectores, dejando espacio suficiente para la posterior incorporación de las calles, obteniéndose layouts más económicos. Es importante la circulación interna de camiones asociada al transporte de sustancias peligrosas, la definición de zonas para carga y descarga, entre otras, como se pueden apreciar en Ponzone et al. (2019). Las distancias mínimas entre las secciones de planta, a excepción de la playa de tanques de metanol, fueron adoptadas de acuerdo a los distanciamientos mínimos establecidos por la secretaría de energía en la resolución 1296/2008. La playa de tanques de metanol fue diseñada de acuerdo al modelo de optimización mencionado y la distancia de impacto es función de este diseño adoptado. A continuación, se desarrolla la metodología propuesta para resolver el problema.

\section{Primera Etapa. Modelo de Optimización de la Playa de Almacenamiento}

En esta etapa se optimiza una función económica (costo) considerando la vulnerabilidad del entorno (radiación límite); a través de la implementación en un modelo MILP, el cual es resuelto en GAMS. Este modelo contiene 1224 variables (920 binarias, 71 discretas y 233 continuas); 9881 restricciones (721 igualdades y 9160 desigualdades) y es resuelto con el solver SCIP en 3 minutos, 15 segundos. El costo de la playa de tanques tiene en cuenta el terreno, el 
endicamiento y los tanques. Si existen tanques embebidos en la llama, se considera, además, el costo de reposición de los mismos (ponderado por su probabilidad de ocurrencia).

El campo de radiaciones en el exterior de la playa de tanques (dada una potencial fuga y posterior incendio del combustible derramado), ha sido calculado a través de un modelo, asegurando estimaciones conservadoras para la determinación del campo de iso-radiación a un nivel especificado. En el modelo de optimización, este campo ha sido aproximado a través de un octágono irregular con el objetivo de considerar el efecto de borde característico del incendio de charcos contenidos/endicados (trench fire) sin perder la linealidad del modelo de optimización (Figura 1). Para mayor detalle del modelo ver Orellano et al. (2019 a).
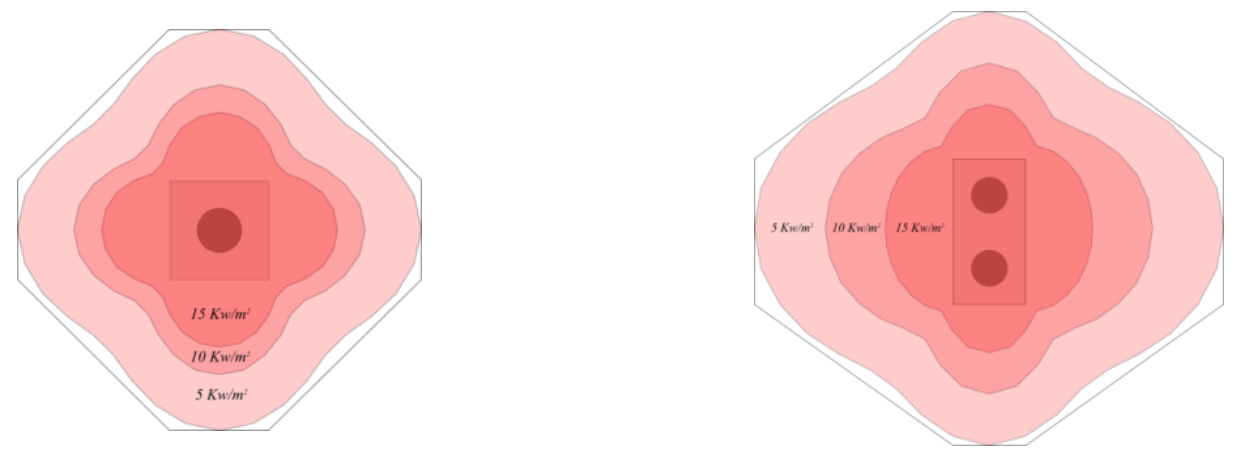

Figura 1. Campos de iso-radiación

La legislación aplicable al almacenamiento de sustancias potencialmente peligrosas establece restricciones que limitan la distancia entre tanques y el volumen del endicamiento que los contiene. La ley 13.660 aplicable a playas de almacenamiento de combustibles, establece en los artículos 321 y 329 estas restricciones, que fueron incluidas en el modelo de optimización.

Dado un volumen de sustancia a almacenar, la estructura de la playa de tanques óptima se deriva de la "superestructura" mostrada en la Figura 2. Esto es, se establecen 16 tanques (subdivisión máxima del inventario total a almacenar) en los cuales podría almacenarse la sustancia adoptando una figura simétrica de no más de 4 tanques en línea. Debe remarcarse que el impacto externo se reduce al dividir el inventario que provoca el incendio, pero aumenta el costo de la instalación de almacenamiento. Se ha definido, para el modelado matemático de la playa de tanques, una variable binaria asociada a cada tanque que indica la presencia o ausencia del mismo. El manejo de las variables binarias puede observarse en detalle en Orellano et al. (2019 a).

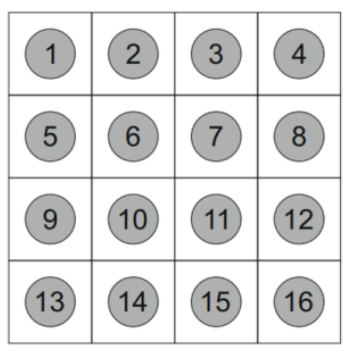

Figura 2. Esquema base

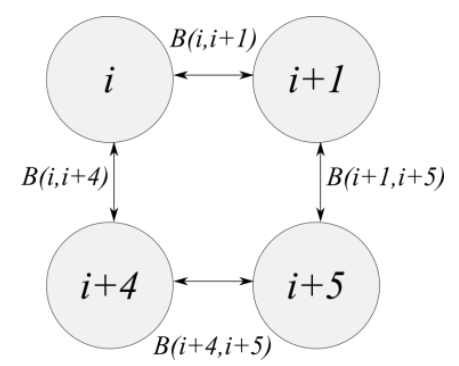

Figura 3. Esquema de grafo

Los tanques se han numerado comenzando desde la esquina superior izquierda, y la presencia de endicamientos se ha modelado a través de un grafo en el que los nodos del mismo representan a los tanques, y los arcos a las paredes del endicamiento presentes entre ambos (Figura 3).

Es preciso mencionar que se ha realizado la simplificación (considerando que los tanques pueden agruparse en distintos endicamientos) forzando que cada endicamiento cuente con el mismo número de tanques. Esto simplifica el problema ya que las dimensiones de cada endicamiento no resultan una función lineal del número de tanques que contiene. Esta, además de simplificar el modelo, representa una disminución de los costos de construcción.

Se ha adoptado el valor de $\mathrm{P}=10^{-4}$ (tanque.año) ${ }^{-1}$ (HSE-UK, 2012), para la tasa de fallas para derrames en tanques con contenido inflamable. Considerando la probabilidad de falla de un tanque independiente de la del resto, entonces la probabilidad de que uno de un conjunto de tanques falle (fuga), es fácilmente calculable. A partir de esto, es posible cuantificar en términos económicos la destrucción provocada, ponderada por la probabilidad de ocurrencia, y su consecuente inclusión en la función objetivo.

En cuanto a la "vulnerabilidad del espacio exterior" de la playa, se utilizó el modelo de radiación previamente mencionado para calcular el área externa sujeta a una radiación superior o igual a la fijada para evitar efecto dominó $\left(15 \mathrm{KW} / \mathrm{m}^{2}, t \geq 10 \mathrm{~min}\right)$. El cálculo del área se realizó a través de la categorización de la misma en tres y la adición de estas tres categorías en la variable $A_{\text {ext. }}$ En el algoritmo llevado a cabo, para evitar la pérdida de generalidad y la 
contabilización por duplicado de las áreas sujetas a niveles de radiación superiores a los fijados causados por más de un frente de llama, se calculó el área externa a través de los siguientes pasos: en primer lugar, se enmarca la playa (cualquiera sea la forma obtenida) en un rectángulo y se calcula el área interior del rectángulo no ocupada por la playa. En segundo lugar, una vez enmarcada la playa, se calcula el área externa normal a los lados, determinando los puntos extremos sobre rectas horizontales y verticales (Fig. 4). Como mencionamos anteriormente, una buena aproximación para los puntos de iso-radiación en los vértices de los endicamientos se puede realizar a través de rectas. Esta área determinada entre los valores extremos horizontales y verticales, representa superficies triangulares, siendo ésta, la última etapa del cálculo.

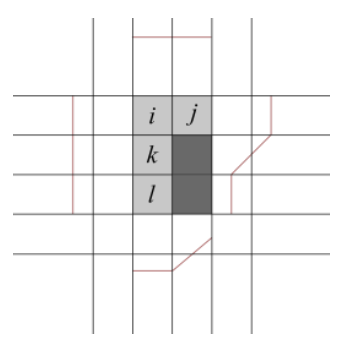

Figura 4. Cálculo del área

La función objetivo incluye el costo (anualizado) de los tanques; del área ocupada (terreno y construcción de la base del endicamiento); costo de construcción de las paredes del endicamiento, de cañerías, el asociado a la vulnerabilidad interna de la playa y el área externa a la playa sujeta a un nivel de radiación superior a $15 \mathrm{Kw} / \mathrm{m}^{2}$. El problema se resuelve paramétricamente en cuanto al número de tanques y la cantidad que se disponen por endicamiento (Orellano et al., 2019 a). El modelo implementado brinda como resultado las distintas disposiciones óptimas (número de tanques y modo en que se agrupan), indicadas en las siguientes figuras. En la Figura 5.(a) se observa el costo óptimo de la disposición de la playa de almacenamiento para distintos números de tanques considerados. Se observa, la presencia de un óptimo para $n_{t o t}: 4$ tanques. La distribución óptima asociada a $n_{t o t}: 4$ se muestra en la Figura 5.(b), observándose la existencia de las paredes intermedias divisoras en el dique (en la solución óptima).

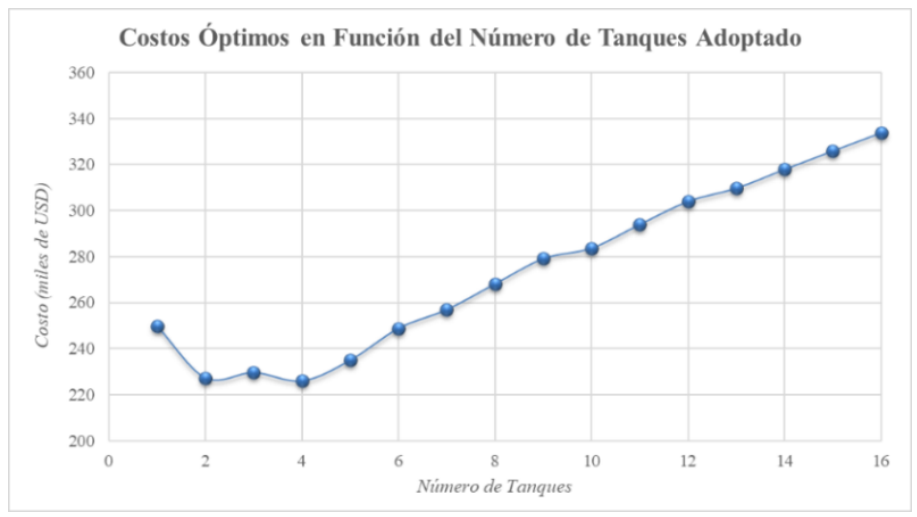

5.(a) Costos óptimos de la playa de tanques de metanol

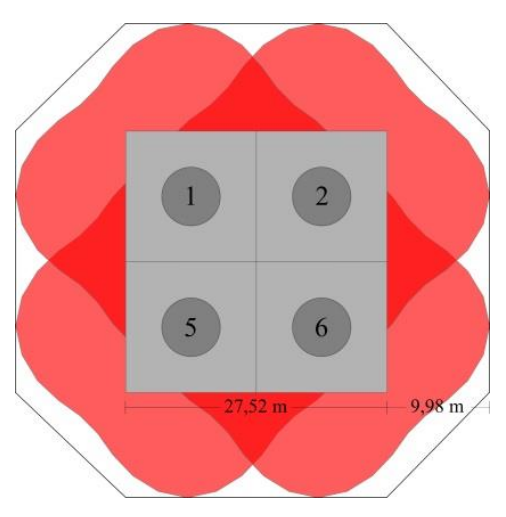

5.(b) Distribución óptima

Figura 5. Resultados

\section{Segunda Etapa. Modelo de Optimización del layout}

La optimización del layout del complejo industrial se realizó a través de la implementación de un modelo MILP, el cual es resuelto en GAMS. Para los datos disponibles en este caso de estudio, y los datos óptimos obtenidos para la playa de almacenamiento en la primera etapa de la optimización, se procede a continuación a resolver el modelo de optimización del layout global. Este modelo contiene 447 variables (189 binarias y 258 continuas); 1061 restricciones (85 igualdades y 976 desigualdades) y es resuelto con el solver SCIP en tiempos inferiores a 19 minutos. Se enumeran a continuación, ciertas particularidades del modelo de optimización:

- Se incorpora la decisión conservadora asumiendo que la distancia mínima establecida entre unidades se cumpla en al menos una de las dos direcciones coordenadas. Esta relajación permite que el modelo no pierda la linealidad, lo que ocurriría al utilizar distancias euclideas. En cuanto a las distancias consideradas desde la playa de almacenamiento de metanol, que es la que mayores distancias de impacto tiene asociada, se ha aproximado la misma con mayor rigurosidad a través de un octágono (de manera similar a lo explicitado para el campo de isoradiación, se ha procedido para la concentración asociada al ERPG-3). A través de esta mejora se logra una 
disminución en los costos (por la ampliación de la región factible) asociado a un aumento en los tiempos de resolución por la incorporación de nuevas variables binarias.

- Se consideran las distancias mínimas requeridas entre los bordes de las instalaciones y los límites del terreno, tal como exige la legislación aplicable a los distintos tipos de plantas. En el caso del metanol, se considera aquella que se corresponde con el nivel de ERPG-3 para la dispersión de tóxicos (según corresponda).

- Se consideró la distancia Manhattan entre las instalaciones para el cálculo de la conectividad. Su utilización busca representar la forma de conectar las unidades en planta.

Las restricciones consideradas incluyen aquellas correspondientes a la definición de los límites del terreno, aquellas cuya finalidad es evitar el solapamiento entre las distintas unidades y el cumplimiento de cierto distanciamiento entre las mismas como mencionamos previamente, entre otras. El modelo se plantea como un MILP a través de la linealización de las restricciones mediante términos de tipo BigM (ver Orellano et al., 2018).

La función objetivo a minimizar corresponde al costo total como se observa en Ec. (1).

$$
\text { Costo }=\text { Costo }_{\text {area }} X Y+\text { Costo }_{\text {conec }}
$$

Siendo, Costo arrea el costo de la unidad de área de terreno y Costo $_{\text {conec }}$ el costo de conectividad, función de la distancia, caudal y condiciones de proceso.

El producto de las longitudes de los lados del terreno $(X Y)$ genera la no linealidad y la no-convexidad de la función objetivo. Este producto bilineal ha sido linealizado a través de la relajación de McCormick. Para realizarlo, se recurrió a la división del dominio original en subdominios, realizado a través de un modelado con decisiones discretas.

\section{Resultados}

Habiendo establecido la distancia de impacto dado el evento incendio, y dado que actualmente no se incluye en el modelo la estimación del impacto por toxicidad, se procede a calcular la distancia de impacto (fuga y dispersión de tóxico). Se realizó la estimación mediante Aloha (EPA, 2016) de la distancia a la cual se manifestaría la concentración correspondiente al ERPG-3 (adoptado como el máximo nivel de daño a la persona tolerado) debido a la difusión de metanol en las condiciones ambientales más adversas (elevada estabilidad atmosférica y una velocidad de viento calmo). Se dispone por lo tanto de las distancias mínimas a verificarse entre la playa óptima de tanques de metanol y las demás, ya sea las de frecuente presencia de personas (oficinas, talleres, sala de control), como las de operaciones.

Se muestran a continuación (Figura 6) los diferentes layouts obtenidos. Por un lado, considerando la existencia de viviendas en el exterior y por lo tanto restringiendo al interior de la planta la zona donde se manifestaría el nivel de ERPG-3 asociado a un derrame de metanol con la consecuente evaporación del charco asociado, asumiendo las peores condiciones ambientales (Figura 6.a) y por otro, sin considerar la vulnerabilidad del entorno a la planta (Figura 6.b). Por definición, el ERPG-3 corresponde a la zona que debiera ser evacuada inmediatamente, por lo que es una restricción razonable imponer que aún en las condiciones ambientales más adversas, este límite se encuentre confinado a los límites de la planta. Obviamente, deberán realizarse planes de evacuación de la planta ante contingencias y eventualmente de la zona que la circunda.

$\begin{array}{ll}\text { Layout (a): } & \text { Costo }_{\text {conectividad }} \text { USD } 64.330 \\ \text { Layout (b): } & \text { Costo } \text { conectividad }_{\text {USD }} 60.696\end{array}$

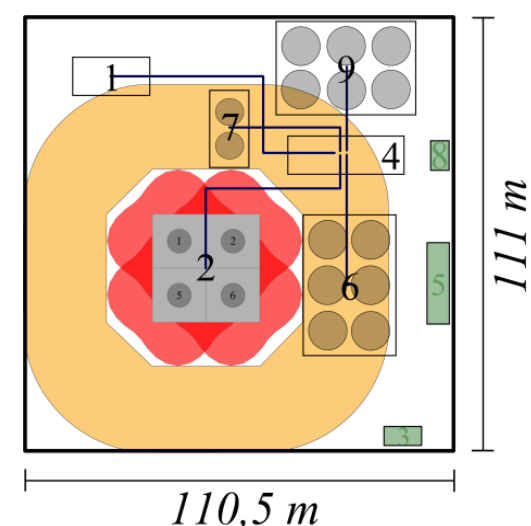

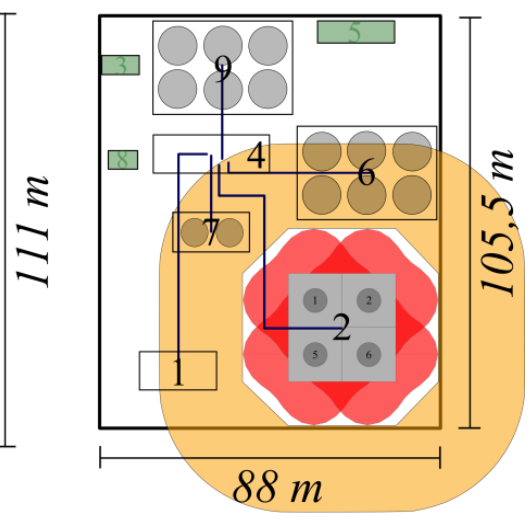

Costo $_{\text {terreno: }}:$ USD 184.051

Costoterreno: USD 139.260

\author{
Sala de calderas (1) \\ Playa de tanques de metanol (2) \\ Oficinas (3) \\ Planta de producción (4) \\ Taller de mantenimiento (5) \\ Playa de tanques de biodiesel (6) \\ Playa de tanques de glicerina (7) \\ Sala de control (8) \\ Playa de tanques de aceite (9)
}

6.(a) Layout óptimo restringiendo el ERPG-3 $\quad$ 6.(b) Layout óptimo sin restringir el ERPG-3

Figura 6. Resultados layouts óptimos

Analizando las soluciones óptimas obtenidas, en cuanto a la playa de tanques de metanol en ambas alternativas, su ubicación es esperable. Por un lado, si no se considera la vulnerabilidad del entorno, la playa se ubica en un extremo dando lugar a más superficie disponible para la ubicación de las unidades frecuentadas por el personal. La zona de proceso se ubica centrada, con respecto a las playas de almacenamiento de modo de reducir el costo de conectividad. 
Por otro lado, si se considera la vulnerabilidad del entorno, el modelo establece la localización de la playa de metanol en el centro de la planta y el resto de las secciones se conforman alrededor de ésta; obviamente la zona correspondiente al proceso también se ubica en una posición centrada con respecto a las secciones de almacenamiento de las otras sustancias. En ambas disposiciones se observa además el alejamiento de la sala de calderas del resto de las unidades; esto es por las restricciones asociadas al riesgo de dicha unidad (distancia de impacto por explosión).

\section{Discusión y Conclusiones}

Existen escasos trabajos y/o metodologías difundidas que permitan en lo posible "conectar" la toma de ciertas decisiones en la etapa de síntesis y diseño del layout considerando el riesgo. Este trabajo, aún con ciertas limitaciones evidentes, entre ellas: el número de unidades de procesamiento/instalaciones consideradas, representando una limitación para las técnicas determinísticas de optimización (limitadas a 12 UP), la optimización por etapas mediante ambos modelos de optimización (que podría llevar a la obtención de óptimos relativos), entre otras; sienta las bases para desarrollar una metodología para la obtención del layout óptimo considerando el diseño basado en riesgos; permitiendo además tomar en cuenta simultáneamente, variables correspondientes a etapas anteriores de diseño (de la ingeniería conceptual). Es necesario destacar que no solo no existe reportada tal metodología, y las herramientas que permitan su ejecución; sino que el modelo utilizado en la primera etapa, y su integración al problema del layout, no han sido aún exploradas según nuestro conocimiento.

Resulta importante entonces, proponer pasos en la dirección del diseño óptimo del layout basado en riesgos. Futuros trabajos deberían incorporar mecanismos para la consideración de un mayor número de unidades de procesamiento, y por otro lado, deberían obtenerse correlaciones (o modelos reducidos) para la estimación de distancias de impacto para todos los potenciales eventos en industrias de proceso. Estos modelos, deberían ser lo suficientemente precisos, ya que de ello depende la bondad de la solución óptima obtenida; y a su vez lo suficientemente sencillos como para ser incorporados en un modelo de optimización, evitando la incorporación de excesivas no linealidades y pérdidas de convexidad.

Es evidente que las decisiones adoptadas en la etapa de síntesis del proceso, playa de tanques, entre otros, impactan directamente en el posterior diseño del layout. A través de este trabajo se incluyen notorios avances tendientes a mitigar la desvinculación existente entre estas etapas de diseño estrechamente relacionadas considerando la filosofía de diseño basado en riesgos.

\section{Referencias}

Casal, J. (2008). Evaluation of the Effects and Consequences of Major Accidents in Industrial Plants. Amsterdam, Netherlands, Elsevier.

EPA (2016). ALOHA (5.4.7). https://www.epa.gov/cameo/aloha-software

Health and Safety Executive (2012). Failure Rate and Event Data for use within Risk Assessments. United Kingdom.

Landucci G., Cozzani V. (2009). "The assessment of the damage probability of storage tanks in domino events triggered by fire". Accident Analysis and Prevention 41: 1206-1215.

National Fire Protection Association. (2010). Large-Loss Fires in the United States-2009. United States, NFPA.

Orellano, S.; Scenna, N. J. (2018). “Optimización de layout considerando el diseño inherentemente seguro”. JIT2018. Rafaela, Argentina, 25 y 26 de octubre.

Orellano, S.; Rodríguez, N.; Scenna, N. J. (2019 a). “Optimización de playas de almacenamiento de sustancias peligrosas frente a escenarios de trench fire". XI CAIQ 2019. Santa Fe, Argentina, 5 al 7 de agosto.

Orellano, S.; Scenna, N. J. (2019 b). “Optimización de layout considerando el diseño basado en riesgos”. JIT2019. Rosario, Argentina, 26 y 27 de septiembre.

Ponzone, D. M.; Orellano, S.; Biscotti P.; Scenna N. J. (2019). “Optimización de layout mediante programación matemática empleando ceibo". XI CAIQ 2019. Santa Fe, Argentina, 5 al 7 de agosto.

Resolución 1296. (2008). Condiciones mínimas que deben cumplir las plantas de elaboración y almacenamiento de biocombustibles en relación a la seguridad. Secretaría de Energía de la Argentina. 